\title{
Univariate and bivariate truncated von Mises distributions
}

\author{
Pablo Fernandez-Gonzalez, Concha Bielza, Pedro Larrañaga \\ Department of Artificial Intelligence, Universidad Politécnica de Madrid
}

\begin{abstract}
In this article we study the univariate and bivariate truncated von Mises distribution, as a generalization of the von Mises distribution (Jupp and Mardia (1989)), (Mardia and Jupp (2000)). This implies the addition of two or four new truncation parameters in the univariate and, bivariate cases, respectively. The results include the definition, properties of the distribution and maximum likelihood estimators for the univariate and bivariate cases. Additionally, the analysis of the bivariate case shows how the conditional distribution is a truncated von Mises distribution, whereas the marginal distribution that generalizes the distribution introduced in Singh (2002). From the viewpoint of applications, we test the distribution with simulated data, as well as with data regarding leaf inclination angles (Bowyer and Danson. (2005)) and dihedral angles in protein chains (Murzin AG (1995)). This research aims to assert this probability distribution as a potential option for modelling or simulating any kind of phenomena where circular distributions are applicable.
\end{abstract}

Key words and phrases: Angular probability distributions, Directional statistics, von Mises distribution, Truncated probability distributions.

\section{Introduction}

The von Mises distribution has received undisputed attention in the field of directional statistics (Jupp and Mardia (1989)) and in other areas like supervised classification (Lopez-Cruz et al. (2013)). Thanks to desirable properties such as its symmetry, mathematical tractability and convergence to the wrapped normal distribution (Mardia and Jupp (2000)) for high concentrations, it is a viable option for many statistical analyses. However, angular phenomena may present constraints on the outcomes that are not properly accounted forby the density function of the von Mises probability distribution. Thus, a truncated distribu- 
tion with the capabilities of the von Mises distribution is strongly suggested. Additionally, this direction has need of development, since there is hardly any literature, and to the best of our knowledge, only one paper (Bistrian and Iakob (2008)), proposes a definition of the truncated von Mises distribution.

In this article, we propose a truncated probability distribution for angular values whose parent distribution is the von Mises distribution. The univariate and bivariate cases of this distribution are explicitly developed.

Section 2 introduces the definition for the univariate case and derives some properties of the distribution, calculates the maximum likelihood estimators of the parameters and studies the distribution moments. Section 3 addresses the definition of the bivariate truncated von Mises, maximum likelihood estimation of the parameters and the definition and study of the conditional and marginal truncated distributions. Section 4 reports the simulation studies applying the above developments and experimentally testing the observed behaviors. Section 5 reports experiments on real datasets containing leaf inclination angles in the univariate case and dihedral angles in protein chains in the bivariate case. Finally, Section 6 discusses the summary and conclusions.

The proofs of all results can be found in the supplementary material.

\section{Univariate truncated von Mises distribution}

Definition 2.1 The truncated von Mises distribution is presented as a fourparameter generalization of the non-truncated case for truncation parameters $a, b$

$a s$

$$
f_{t v M}(\theta ; \mu, \kappa, a, b)= \begin{cases}\frac{e^{\kappa \cos (\theta-\mu)}}{\int_{a}^{b} e^{\kappa \cos (\theta-\mu)} d \theta} & \text { if } \theta \in \mathbb{O}_{a, b} \\ 0 & \text { if } \theta \in \mathbb{O}_{b, a}\end{cases}
$$

where $\mu \in \mathbb{O}$ is the location parameter, $\kappa>0$ the concentration parameter, $\mathbb{O}$ is the circular set of points $\left(\mathbb{O}:(x, y)\right.$ such that $\left.x^{2}+y^{2}=1\right), \mathbb{O}_{a, b} \subset \mathbb{O}$ is obtained by selecting the points in the circular path from $a \in \mathbb{O}$ to $b \in \mathbb{O}$ in the preferred 
direction (counterclockwise) and $\mathbb{O}_{b, a}$ is its counterpart w.r.t. $\mathbb{O}$. Our proposed definition differs from Bistrian and Iakob (2008) in the circular definition of the truncation parameters. In their article, the truncation parameters were bounded to a linear definition involving the location parameter. Thanks to this difference, our distribution only needs truncation parameter values contained in $[0,2 \pi]$ in order to represent all possible distributions. This also affects the calculations involving the truncation parameters.

Truncation parameters have a big influence on the shape of the truncated distribution (Figure 1).

Lemma 2.1 $\exists a, b, \mu \in \mathbb{O}$ such that in $\mathbb{O}_{a, b}$ :

1. $f_{t v M}(\theta ; \mu, \kappa, a, b)$ is a strictly decreasing function.

2. $f_{t v M}(\theta ; \mu, \kappa, a, b)$ is a strictly increasing function.

3. $f_{t v M}(\theta ; \mu, \kappa, a, b)$ increases and decreases reaching a single critical point that is a maximum.

4. $f_{t v M}(\theta ; \mu, \kappa, a, b)$ increases and decreases reaching a single critical point that is a minimum.

5. $f_{t v M}(\theta ; \mu, \kappa, a, b)$ increases and decreases reaching two critical points, a maximum and a minimum. 


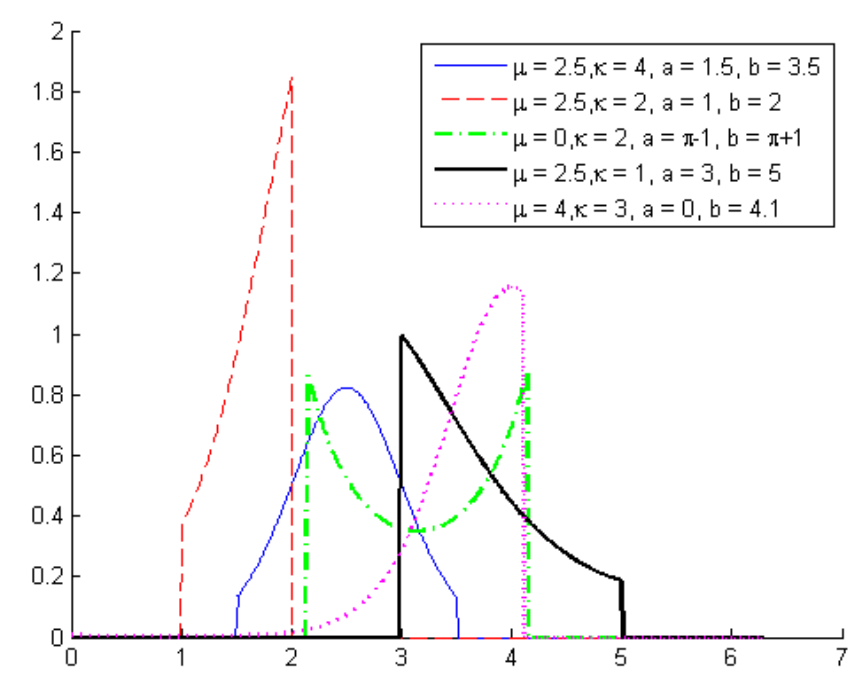

Figure 1: Several truncated von Mises distributions that include all cases of Lemma 2.1. Symmetrical function with maxima not at the extrema (thin continuous line), strictly increasing function (dashed line), strictly decreasing function (thick continuous line), unique critical point that is a minimum (dash-dot line) and two critical points, a maximum and a minimum (dotted line).

Looking at the normalization constant, we find that given $f_{v M}(\theta ; \mu, \kappa)$ and $f_{t v M}(\theta ; \mu, \kappa, a, b)$ such that $\mathbb{O}_{b, a} \neq \varnothing$ then $f_{v M}(\theta)<f_{t v M}(\theta), \quad \forall \theta \in \mathbb{O}_{a, b}$, as would be expected from a truncated distribution. Note then that while truncation parameters are circular quantities, the values for the integration coefficients are linear. Therefore, we use $b+2 \pi$ if $b<a$ as integration coefficients and $a, b$ if $a \leq b$ (Unless otherwise stated, this will be assumed and not written explicitly throughout).

It is a well-known result (Abramowitz and Stegun (1964)) that $2 \pi I_{0}(\kappa)=$ $\int_{0}^{2 \pi} e^{\kappa \cos (\theta-\mu)} d \theta$, where $I_{0}(\kappa)$ is the modified Bessel function of the first kind and order 0 , that is,

$$
I_{0}(\kappa)=\sum_{m=0}^{\infty} \frac{x^{2 m}}{(m !)^{2} 2^{m}} .
$$

The above expression suffices for truncation parameters $a, b$ such that $\mathbb{O}_{a, b}=\mathbb{O}$. However, it is necessary to calculate the general case for non-restricted truncation parameters. Taking $w=\left\lfloor\frac{n}{2}\right\rfloor+\bmod \frac{n}{2}-1$, we obtain: 
Lemma 2.2 $\int_{a}^{b} e^{\kappa \cos (\theta-\mu)} d \theta=I(b ; \mu, \kappa)-I(a ; \mu, \kappa)$, where

$$
\begin{aligned}
I(\theta ; \mu, \kappa)= & \sum_{n=0}^{\infty} \frac{\kappa^{n}}{n !}\left(\sin (\theta-\mu) \sum_{i=0}^{w}\left(\cos ^{n-2 i-1}(\theta-\mu) \prod_{j=0}^{2 i}(n-j)^{-(-1)^{j}}\right)\right. \\
& \left.+\frac{\left((-1)^{n}+1\right) \prod_{j=0}^{w}(n-j)^{-(-1)^{j}}(\theta-\mu)}{2}\right) .
\end{aligned}
$$

$I(\theta ; \mu, \kappa)$ is the distribution function of the positive support of the truncated von Mises density.

\subsection{Maximum likelihood estimation}

Provided we have a sample of observations $\theta_{1}, \theta_{2}, \ldots, \theta_{n}$ from a truncated von Mises distribution (1), we obtain:

$$
\begin{aligned}
\ln L\left(\mu, \kappa, a, b ; \theta_{1}, \theta_{2}, \ldots, \theta_{n}\right) & =\sum_{i=1}^{n} \ln \left(\frac{e^{\kappa \cos \left(\theta_{i}-\mu\right)}}{\int_{a}^{b} e^{\kappa \cos (\theta-\mu)} d \theta}\right) \\
& =\sum_{i=1}^{n} \kappa \cos \left(\theta_{i}-\mu\right)-n \ln \left(\int_{a}^{b} e^{\kappa \cos (\theta-\mu)} d \theta\right)
\end{aligned}
$$

where $\ln L\left(\mu, \kappa, a, b ; \theta_{1}, \theta_{2}, \ldots, \theta_{n}\right)$ is the log-likelihood function for the truncated von Mises distribution.

We now seek to solve the system of four log-likelihood equations created by the four parameters of the distribution. For parameters $\mu, \kappa$, we have

$$
\begin{gathered}
\frac{\partial \ln L}{\partial \mu}=0 \\
\frac{\partial \ln L}{\partial \kappa}=0 .
\end{gathered}
$$

As parameters $a, b$, define the region of the greater-than-zero density, we find that all $\theta_{1}, \ldots, \theta_{n}$ observations necessarily lie within the subset $\mathbb{O}_{a, b}$. Thanks to this consideration, together with the $-n \ln \left(\int_{a}^{b} e^{\kappa \cos (\theta-\mu)} d \theta\right)$ sub term of (3), i.e., the integral of a solely positive function as the argument of the strictly increasing logarithmic function and $n \in \mathbb{N}$, we can isolate the estimators 


$$
\mathbb{O}_{\hat{a}, \hat{b}}=\underset{a, b}{\operatorname{argmax}}\left(\max \left(\left\{A\left(\mathbb{O}_{\theta_{1}^{\prime}, \theta_{2}^{\prime}}\right), \ldots, A\left(\mathbb{O}_{\theta_{n-1}^{\prime}, \theta_{n}^{\prime}}\right), A\left(\mathbb{O}_{\theta_{n}^{\prime}, \theta_{1}^{\prime}}\right)\right\}\right)\right),
$$

where $A\left(\mathbb{O}_{\theta_{1}, \theta_{2}}\right)$ is the angle between the point with angle $\theta_{1}$ w.r.t. 0 and the point with angle $\theta_{2}$ w.r.t. 0 , and $\left\{\theta_{1}^{\prime}, \ldots, \theta_{n}^{\prime}\right\}$ is the sample sorted in ascending order of value. Intuitively, the truncation parameters are separated by the largest angle and are contiguous in a sorted finite circular sample. Notice that we input the angle between the last and the first element in the sample in order to complete the circle. Consequently, every truncated distribution with truncation parameters whose positive support does not include $0^{\circ}$ maximizes the integral subterm of (3) by means of this inclusion in (4).

From this result, we can say that the truncation parameters of the truncated von Mises distribution have existing and population-only dependent maximum likelihood estimators. For parameters $\mu$ and $\kappa$, interdependency is a consequence of the possibly non-symmetrical shape of the distribution. If we observe the expression of the partial derivatives

$$
\begin{aligned}
\frac{1}{n} \sum_{i=1}^{n} \sin \left(\theta_{i}-\mu\right)-\frac{e^{\kappa \cos (a-\mu)}-e^{\kappa \cos (b-\mu)}}{\int_{a}^{b} e^{\kappa \cos (\theta-\mu)} d \theta} & =0 \\
\frac{1}{n} \sum_{i=1}^{n} \cos \left(\theta_{i}-\mu\right)-\frac{\int_{a}^{b} \cos (\theta-\mu) e^{\kappa \cos (\theta-\mu)} d \theta}{\int_{a}^{b} e^{\kappa \cos (\theta-\mu)} d \theta} & =0
\end{aligned}
$$

$e^{\kappa \cos (a-\mu)}-e^{\kappa \cos (b-\mu)}=0$ holds jf $a, b$ are symmetrical w.r.t. $\mu$, reducing the location parameter estimator to that of the non-truncated case (Mardia and Jupp (2000)), the circular sample mean $\hat{\mu}$. As no population-only dependent expressions of the parameters $\mu$ and $\kappa$ were found, we use optimization techniques to maximize the log-likelihood function for those parameters in our study. To be precise, we regard the optimization of $\mu$ and $\kappa$ as a non-linear programming problem that we can solve as a system of Karush-Kuhn-Tucker conditions. 


\subsection{Moments}

The moments in circular statistics are particular values of the characteristic function. The r-th moment about a direction $d$ can be written as

$$
m_{r_{t v M}}=\mathbb{E}\left[e^{i r(X-d)}\right]
$$

The first moment about the 0 direction for the truncated von Mises is calculated as

$$
m_{1_{t v M}}=\frac{\int_{a}^{b} \cos (\theta) e^{\kappa \cos (\theta-\mu)} d \theta}{\int_{a}^{b} e^{\kappa \cos (\theta-\mu)} d \theta}+\frac{i \int_{a}^{b} \sin (\theta) e^{\kappa \cos (\theta-\mu)} d \theta}{\int_{a}^{b} e^{\kappa \cos (\theta-\mu)} d \theta},
$$

and we can relate (5) to the first moment about the $\mu$ direction, denoted as $m_{1_{t v M}}^{\prime}$ as

$$
m_{1_{t v M}}=e^{i \mu} m_{1_{t v M}}^{\prime} .
$$

Notice that if $\cos (a-\mu)=\cos (b-\mu)$, then $m_{1_{t v M}}^{\prime}=\frac{\int_{a}^{b} \cos (x-\mu) e^{\kappa \cos (x-\mu)} d \theta}{\int_{a}^{b} e^{\kappa \cos (x-\mu)} d \theta}=R$, the mean resultant length of $\mu$ and thus $m_{1_{t v M}}=e^{i \mu} R$.

An alternative expression for $m_{1_{t v}}$ can be found by considering equations $\mathbb{E}[\cos (x)]=R^{\prime} \cos \left(\mu^{\prime}\right)$ and $\mathbb{E}[\sin (x)]=R^{\prime} \sin \left(\mu^{\prime}\right)$, where $R^{\prime}$ and $\mu^{\prime}$ are the sample mean resultant length and sample mean, respectively. We can then state

$$
m_{1_{t v M}}=\mathbb{E}[\cos (x)]+i \mathbb{E}[\sin (x)]=R^{\prime} \cos \left(\mu^{\prime}\right)+i R^{\prime} \sin \left(\mu^{\prime}\right)=R^{\prime} e^{i \mu^{\prime}}
$$

Thus, merging Equations (6) and (7), we obtain

$$
e^{i\left(\mu^{\prime}-\mu\right)} R^{\prime}=m_{1_{t v M}^{\prime}}^{\prime}
$$

which can be seen as a valuable expression as it contains the sample mean $\left(\mu^{\prime}\right)$ and the location parameter of the distribution $(\mu)$.

\section{Bivariate truncated von Mises distribution}

The non-truncated bivariate von Mises distribution was first proposed by Singh (2002) and extended and developed in Mardia et al. (2008) and Mardia and Voss 
(2011). It is a unimodal/bi-modal function on the torus $f_{b t v M}: \mathbb{O} \times \mathbb{O} \rightarrow \mathbb{R}$ obtained by replacing the quadratic and linear terms of the normal bivariate distribution with their circular analogues. This distribution is known as the "sin variant bivariate von Mises distribution" and is defined for dependent pairs of angular variables. It is expressed for variables $\theta_{1}$ and $\theta_{2}$, as

$$
f\left(\theta_{1}, \theta_{2}\right)=C e^{\kappa_{1} \cos \left(\theta_{1}-\mu_{1}\right)+\kappa_{2} \cos \left(\theta_{2}-\mu_{2}\right)+\lambda \sin \left(\theta_{1}-\mu_{1}\right) \sin \left(\theta_{2}-\mu_{2}\right)},
$$

where $\kappa_{1}, \kappa_{2} \geq 0, \lambda \in \mathbb{R}, \mu_{1}, \mu_{2} \in \mathbb{O}$ and $C$ is the normalization constant.

We propose the density function for the truncated case as a nine-parameter function with density defined as follows:

Definition 3.1 We wite the density function for the truncated case as a nineparameter function with density

$$
f_{b t v M}\left(\theta_{1}, \theta_{2} ; \boldsymbol{W}\right)=\left\{\begin{array}{lc}
\frac{f_{u b v M}\left(\theta_{1}, \theta_{2} ; \boldsymbol{W}\right)}{\int_{a_{1} \int_{a_{2}}^{b_{2}} f_{u b v M}\left(\theta_{1}, \theta_{2} ; \boldsymbol{W}\right) d \theta_{2} d \theta_{1}}} & \\
& \text { if } \theta_{1} \in \mathbb{O}_{a_{1}, b_{1}}, \theta_{2} \in \mathbb{O}_{a_{2}, b_{2}}, \\
0 & \text { otherwise }
\end{array}\right.
$$

where $\boldsymbol{W}=\left\{\lambda, \mu_{1}, \mu_{2}, \kappa_{1}, \kappa_{2}, a_{1}, b_{1}, a_{2}, b_{2}\right\}$ is the parameter vector and $f_{u b v M}\left(\theta_{1}, \theta_{2} ; \boldsymbol{W}\right)=e^{\kappa_{1} \cos \left(\theta_{1}-\mu_{1}\right)+\kappa_{2} \cos \left(\theta_{2}-\mu_{2}\right)+\lambda \sin \left(\theta_{1}-\mu_{1}\right) \sin \left(\theta_{2}-\mu_{2}\right)}$ is the unnormalized bivariate von Mises distribution. Parameters $\mu_{1}, \mu_{2}$ and $\kappa_{1}, \kappa_{2}$ are analogous to parameters $\mu$ and $\kappa$, respectively, in the univariate truncated case. Truncation parameters $a_{1}, b_{1}, a_{2}$ and $b_{2}$ are similar to the univariate truncation parameters. The $\lambda \in \mathbb{R}$ parameter accounts for the dependency between the variable components (Figure 2). If $\lambda=0$, then $\theta_{1}$ and $\theta_{2}$ are independent and each is distributed as a univariate von Mises distribution. Also, if $\theta_{1}, \theta_{2}$ are independent, then $\lambda=0$. 


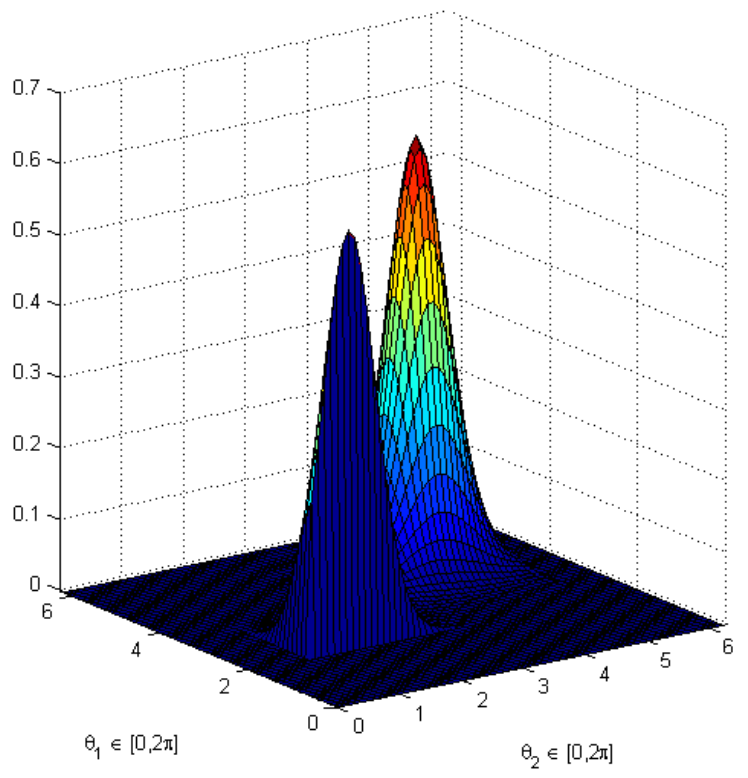

Figure 2: Example of the bi-dimensional von Mises distribution with parameters $\lambda=$ $6, \mu_{1}=\pi, \mu_{2}=3, \kappa_{1}=2, \kappa_{2}=1, a_{1}=2, b_{1}=6, a_{2}=1, b_{2}=5.5$, showing truncated bimodality.

A desirable property of a joint distribution is that it should have closed distributions under marginalization and conditioning, i.e., the marginal and conditional distributions should also follow the univariate distribution. Particularizing for the von Mises family, the bivariate von Mises distribution presents closed distributions only under conditioning as shown by Singh (Singh (2002)). We want to find out whether this also holds for the truncated case.

\subsection{Maximum Likelihood Estimation}

The maximum likelihood estimator for the bivariate distribution takes data of the form $\left\{\left(\theta_{1 i}, \theta_{2 i}\right)\right\} i=1, \ldots, n$. The resulting log-likelihood function is 


$$
\begin{aligned}
& \ln L\left(\boldsymbol{W} ;\left(\theta_{11}, \theta_{21}\right), \ldots,\left(\theta_{1 n}, \theta_{2 n}\right)\right) \\
= & \sum_{i=1}^{n} \ln \left(\frac{e^{\kappa_{1} \cos \left(\theta_{1 i}-\mu_{1}\right)+\kappa_{2} \cos \left(\theta_{2 i}-\mu_{2}\right)+\lambda \sin \left(\theta_{1 i}-\mu_{1}\right) \sin \left(\theta_{2 i}-\mu_{2}\right)}}{\int_{a_{1}}^{b_{1}} \int_{a_{2}}^{b_{2}} e^{\kappa_{1} \cos \left(\theta_{1}-\mu_{1}\right)+\kappa_{2} \cos \left(\theta_{2}-\mu_{2}\right)+\lambda \sin \left(\theta_{1}-\mu_{1}\right) \sin \left(\theta_{2}-\mu_{2}\right)} d \theta_{2} d \theta_{1}}\right) \\
= & \sum_{i=1}^{n}\left(\kappa_{1} \cos \left(\theta_{1 i}-\mu_{1}\right)+\kappa_{2} \cos \left(\theta_{2 i}-\mu_{2}\right)+\lambda \sin \left(\theta_{1 i}-\mu_{1}\right) \sin \left(\theta_{2 i}-\mu_{2}\right)\right) \\
& -n \ln \left(\int_{a_{1}}^{b_{1}} \int_{a_{2}}^{b_{2}} e^{\kappa_{1} \cos \left(\theta_{1}-\mu_{1}\right)+\kappa_{2} \cos \left(\theta_{2}-\mu_{2}\right)+\lambda \sin \left(\theta_{1}-\mu_{1}\right) \sin \left(\theta_{2}-\mu_{2}\right)} d \theta_{2} d \theta_{1}\right) .
\end{aligned}
$$

Thus we have

$$
\frac{\partial}{\partial \mu_{1}} \ln L\left(\boldsymbol{W} ;\left(\theta_{11}, \theta_{21}\right), \ldots,\left(\theta_{1 n}, \theta_{2 n}\right)\right)=0
$$

that is,

$$
\sum_{i=1}^{n} \kappa_{1} \sin \left(\theta_{1 i}-\mu_{1}\right)-\lambda \cos \left(\theta_{1 i}-\mu_{1}\right) \sin \left(\theta_{2 i}-\mu_{2}\right)-\frac{n\left(\int_{a_{2}}^{b_{2}} f_{u b v M}\left(a_{1}, \theta_{2}\right)-f_{u b v M}\left(b_{1}, \theta_{2}\right) d \theta_{2}\right)}{\int_{a_{1}}^{b_{1}} \int_{a_{2}}^{b_{2}} f_{u b v M}\left(\theta_{1}, \theta_{2}\right) d \theta_{2} d \theta_{1}}=0
$$

where $f_{u b v M}\left(\theta_{1}, \theta_{2}\right)$ is the following unnormalized bivariate truncated von Mises function

$$
f_{u b v M}\left(\theta_{1}, \theta_{2}\right)=e^{\kappa_{1} \cos \left(\theta_{1}-\mu_{1}\right)+\kappa_{2} \cos \left(\theta_{2}-\mu_{2}\right)+\lambda \sin \left(\theta_{1}-\mu_{1}\right) \sin \left(\theta_{2}-\mu_{2}\right)} .
$$

Similarly, the partial derivate w.r.t. $\mu_{2}$ gives

$$
\sum_{i=1}^{n} \kappa_{2} \sin \left(\theta_{2 i}-\mu_{2}\right)-\lambda \cos \left(\theta_{2 i}-\mu_{2}\right) \sin \left(\theta_{1 i}-\mu_{1}\right)-\frac{n\left(\int_{a_{1}}^{b_{1}} f_{u b v M}\left(\theta_{1}, a_{2}\right)-f_{u b v M}\left(\theta_{1}, b_{2}\right) d \theta_{1}\right)}{\int_{a_{1}}^{b_{1}} \int_{a_{2}}^{b_{2}} f_{u b v M}\left(\theta_{1}, \theta_{2}\right) d \theta_{2} d \theta_{1}}=0 .
$$

For $\kappa_{1}$ we have

$$
\frac{\partial}{\partial \kappa_{1}} \ln L\left(\boldsymbol{W} ;\left(\theta_{11}, \theta_{21}\right), \ldots,\left(\theta_{1 n}, \theta_{2 n}\right)\right)=0
$$

that is,

$$
\frac{1}{n} \sum_{i=1}^{n} \cos \left(\theta_{1 i}-\mu_{1}\right)-\frac{\int_{a_{1}}^{b_{1}} \int_{a_{2}}^{b_{2}} \cos \left(\theta_{1}-\mu_{1}\right) f_{u b v M}\left(\theta_{1}, \theta_{2}\right) d \theta_{2} d \theta_{1}}{\int_{a_{1}}^{b_{1}} \int_{a_{2}}^{b_{2}} f_{u b v M}\left(\theta_{1}, \theta_{2}\right) d \theta_{2} d \theta_{1}}=0
$$


Similarly, the partial derivate w.r.t. $\kappa_{2}$ gives

$$
\frac{1}{n} \sum_{i=1}^{n} \cos \left(\theta_{2 i}-\mu_{2}\right)-\frac{\int_{a_{1}}^{b_{1}} \int_{a_{2}}^{b_{2}} \cos \left(\theta_{2}-\mu_{2}\right) f_{u b v M}\left(\theta_{1}, \theta_{2}\right) d \theta_{2} d \theta_{1}}{\int_{a_{1}}^{b_{1}} \int_{a_{2}}^{b_{2}} f_{u b v M}\left(\theta_{1}, \theta_{2}\right) d \theta_{2} d \theta_{1}}=0 .
$$

At this point, we can see that both equations (9) and (10), involving $\kappa_{1}, \kappa_{2}$ parameters, respectively, preserve their analogy with the univariate case. Their second addend corresponds to the definition of the estimators of $\mathbb{E}\left[\cos \left(\theta_{1}\right.\right.$ $\left.\left.\mu_{1}\right)\right]$ and $\mathbb{E}\left[\cos \left(\theta_{2}-\mu_{2}\right)\right]$, respectively.

For the parameter $\lambda$ we obtain

$$
\frac{\partial}{\partial \lambda} \ln L\left(\boldsymbol{W} ;\left(\theta_{11}, \theta_{21}\right), \ldots,\left(\theta_{1 n}, \theta_{2 n}\right)\right)=0
$$

that is,

$\frac{1}{n} \sum_{i=1}^{n} \sin \left(\theta_{1 i}-\mu_{1}\right) \sin \left(\theta_{2 i}-\mu_{2}\right)-\frac{\int_{a_{1}}^{b_{1}} \int_{a_{2}}^{b_{2}} \sin \left(\theta_{1}-\mu_{1}\right) \sin \left(\theta_{2}-\mu_{2}\right) f_{u b v M}\left(\theta_{1}, \theta_{2}\right) d \theta_{2} d \theta_{1}}{\int_{a_{1}}^{b_{1}} \int_{a_{2}}^{b_{2}} f_{u b v M}\left(\theta_{1}, \theta_{2}\right) d \theta_{2} d \theta_{1}}=0$,

which analogously corresponds to the estimator of $\mathbb{E}\left[\sin \left(\theta_{1}-\mu_{1}\right) \sin \left(\theta_{2}-\mu_{2}\right)\right]$.

As in the univariate case, the truncation parameters has the following isolated estimators

$$
\begin{aligned}
& \mathbb{O}_{\hat{a}_{1}, \hat{b}_{1}}=\underset{a_{1}, b_{1}}{\operatorname{argmax}}\left(\max \left(\left\{A\left(\mathbb{O}_{\theta_{11}^{\prime}, \theta_{12}^{\prime}}\right), \ldots, A\left(\mathbb{O}_{\theta_{1 n-1}^{\prime}, \theta_{1 n}^{\prime}}\right), A\left(\mathbb{O}_{\theta_{1 n}^{\prime}, \theta_{11}^{\prime}}\right)\right\}\right)\right) \\
& \mathbb{O}_{\hat{a}_{2}, \hat{b}_{2}}=\underset{a_{2}, b_{2}}{\operatorname{argmax}}\left(\max \left(\left\{A\left(\mathbb{O}_{\theta_{21}^{\prime}, \theta_{22}^{\prime}}\right), \ldots, A\left(\mathbb{O}_{\theta_{2 n-1}^{\prime}, \theta_{2 n}^{\prime}}\right), A\left(\mathbb{O}_{\theta_{2 n}^{\prime}, \theta_{21}^{\prime}}\right)\right\}\right)\right),
\end{aligned}
$$

while as yielded by the above calculations, the expressions regarding the nontruncation parameters enhibit interdependency. We optimize them as a nonlinear programming problem in the form of a system of Karush-Kuhn-Tucker conditions, as we did in univariate case.

\subsection{Conditional truncated von Mises distribution}

The density of the conditional truncated von Mises distribution is defined as:

Definition 3.2 The conditional truncated von Mises distribution has density

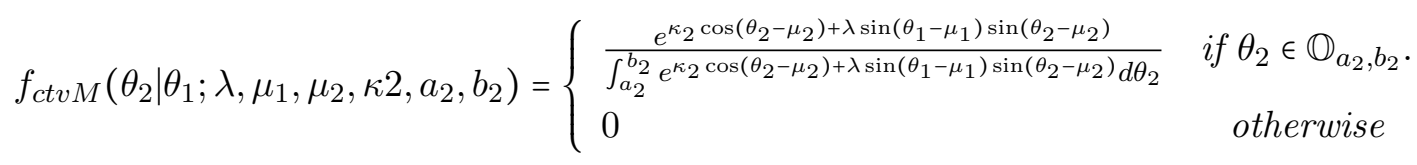


It is a six-parameter distribution where the parameters hold the same meaning as in the bivariate case, with the simplification of parameters $\kappa_{1}, a_{1}, b_{1}$ for $f_{c t v M}\left(\theta_{2} \mid \theta_{1}\right)$ (or $\kappa_{2}, a_{2}, b_{2}$ for $f_{c t v M}\left(\theta_{1} \mid \theta_{2}\right)$ ). Worthy of note, however, is that $\theta_{1} \in \mathbb{O}_{a_{1}, b_{1}}$ in $f_{c t v M}\left(\theta_{2} \mid \theta_{1}\right)$ since otherwise, by the definition of the conditional distribution $f_{c t v M}\left(\theta_{2} \mid \theta_{1}\right)=\frac{f_{b t v M}\left(\theta_{2}, \theta_{1}\right)}{f_{t v M}\left(\theta_{1}\right)}, f_{c t v M}\left(\theta_{2} \mid \theta_{1}\right)$ is not defined.

Theorem 3.1 A conditional truncated von Mises distribution corresponds to a univariate truncated von Mises distribution through

$$
\begin{gathered}
f_{c t v M}\left(\theta_{2} \mid \theta_{1} ; \lambda, \mu_{1}, \mu_{2}, \kappa_{2}, a_{2}, b_{2}\right)= \\
f_{t v M}\left(\theta_{2} ; \mu_{2}+\arctan \left(\frac{\lambda \sin \left(\theta_{1}-\mu_{1}\right)}{\kappa_{2}}\right), \sqrt{\kappa_{2}^{2}+\left(\lambda \sin \left(\theta_{1}-\mu_{1}\right)\right)^{2}}, a_{2}, b_{2}\right),
\end{gathered}
$$

which completely specifies the behavior and properties of the conditional distribution and is analogous to the non-truncated conditional case (Singh (2002)).

\subsection{Marginal truncated von Mises distribution}

We can define the density function of the marginal truncated von Mises distribution as:

Definition 3.3 The density function of the marginal truncated von Mises distribution can be written as

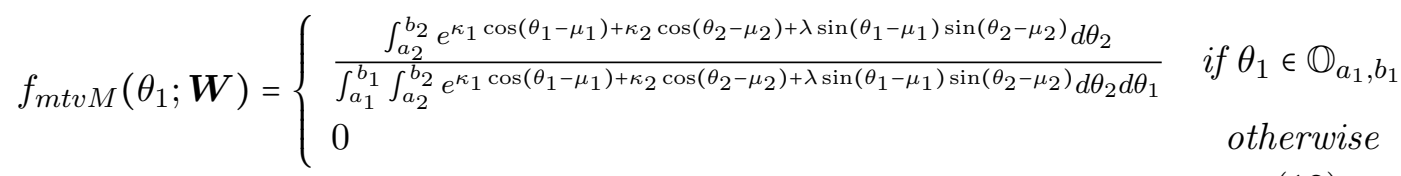

It is a nine-parameter distribution that shares all the parameters with the bivariate truncated von Mises distribution. In the original publication, Singh (2002) studied the distribution and reported the "frontiers" of bi-modality (for $\mu=0$ ) as

$$
\frac{I_{1}\left(\kappa_{2}\right)}{I_{0}\left(\kappa_{2}\right)}=\frac{\kappa_{1} \kappa_{2}}{\lambda^{2}}
$$

where the distribution is unimodal if $\frac{I_{1}\left(\kappa_{2}\right)}{I_{0}\left(\kappa_{2}\right)} \geq \frac{\kappa_{1} \kappa_{2}}{\lambda^{2}}$, and bimodal with two equal maxima otherwise. Additionally, the modes were calculated to be symmetrical 
w.r.t $\mu_{1}$ and at the distance value $\theta_{1}^{*}$ that solves the equation (for $\mu_{1}=0$ ):

$$
\frac{A\left(\sqrt{\kappa_{2}+\lambda^{2} \sin ^{2}\left(\theta_{1}^{*}\right)}\right)}{\sqrt{\kappa_{2}+\lambda^{2} \sin ^{2}\left(\theta_{1}^{*}\right)}} \cos \left(\theta_{1}^{*}\right)=\frac{\kappa_{1}}{\lambda^{2}},
$$

where $A(x)=\frac{I_{1}(x)}{I_{0}(x)}$. In order to generalize this analysis to cover the truncated case in (12), we need to account for the contribution made by the parameters $\mu_{2}, a_{2}$ and $b_{2}$ to the shape of the distribution. $\mu_{2}$ is not necessarily simplified by the symmetry of $a_{2}$ and $b_{2}$ in the non-truncated case. Additionally, $a_{2}$ and $b_{2}$ do not behave as truncation parameters, as they do not address the argument of the function and appear in both the numerator of the expression and the normalization term, actively influencing the resultant shape of the proposed distribution. Contrary to the non-truncated case, a truncated marginal distribution that exhibits two maxima may have only one global maximum; and. even if it has only one maximum, the distribution is not necessarily centered around the mean (Figure 3). Therefore, our analysis determines the different parameter configurations that produce the whole range of behaviors, with a special focus on bi-modality/unimodality results. Parameters $a_{1}$ and $b_{1}$ behave as the truncation parameters studied for the univariate case. 


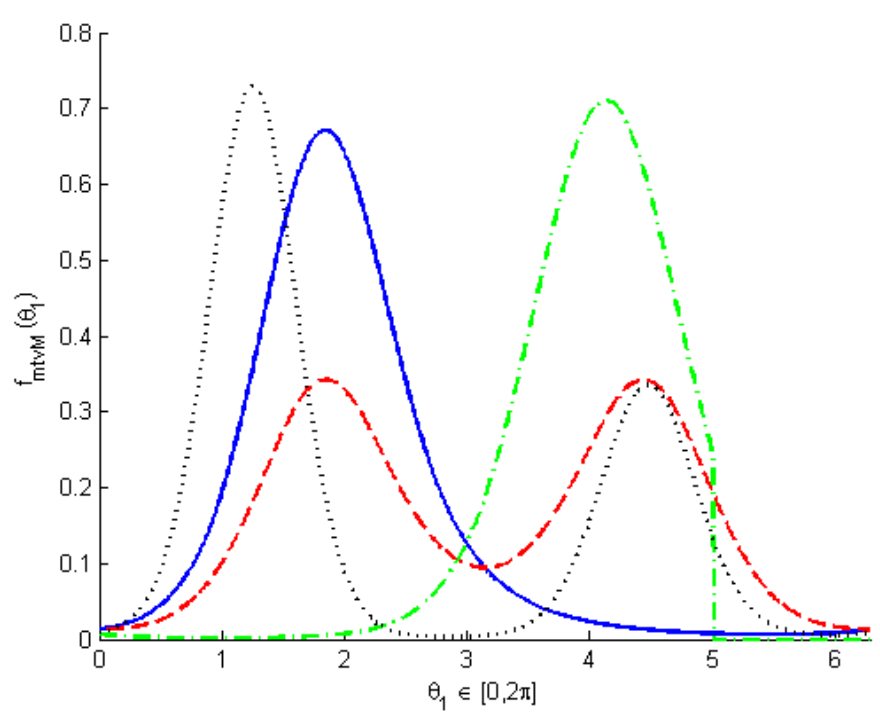

Figure 3: Several truncated marginal distributions showing unimodality (continuous line) with parameters $\lambda=5, \mu_{1}=\pi, \mu_{2}=0, \kappa_{1}=1, \kappa_{2}=4, a_{1}=0, b_{1}=2 \pi, a_{2}=\pi-0.2, b_{2}=2 \pi$, two equal maxima (dashed line) with parameters $\lambda=5, \mu_{1}=\pi, \mu_{2}=0, \kappa_{1}=1, \kappa_{2}=$ $4, a_{1}=0, b_{1}=2 \pi, a_{2}=0, b_{2}=2 \pi$, truncated unimodality (dash-dot line) with parameters $\lambda=1, \mu_{1}=4, \mu_{2}=2, \kappa_{1}=3, \kappa_{2}=4, a_{1}=0, b_{1}=5, a_{2}=2, b_{2}=2 \pi$ and two distinct maxima (dotted line) with parameters $\lambda=10, \mu_{1}=6, \mu_{2}=1, \kappa_{1}=0.3, \kappa_{2}=6, a_{1}=0, b_{1}=2 \pi, a_{2}=$ $0, b_{2}=5$ respectively.

If, without loss of generality, we take $\theta_{1^{\prime}}=\theta_{1}-\mu_{1}$, we can postulate the following theorem:

Theorem 3.2 All different behaviors w.r.t. the unimodality/bi-modality of the marginal truncated von Mises distribution can be accounted for as follows

1. $f_{m t v M}\left(\theta_{1^{\prime}}\right)$ is unimodal with mode (maximum) in $\mu_{1}$, if and only if $T\left(\lambda, \mu_{2}, \kappa_{1}, \kappa_{2}, a_{2}, b_{2}\right)<0$ and $\cos \left(b_{2}-\mu_{2}\right)=\cos \left(a_{2}-\mu_{2}\right)$.

2. $f_{m t v M}\left(\theta_{1^{\prime}}\right)$ is bi-modal with equal maxima, if and only if $T\left(\lambda, \mu_{2}, \kappa_{1}, \kappa_{2}, a_{2}, b_{2}\right)>$ 0 and $\cos \left(b_{2}-\mu_{2}\right)=\cos \left(a_{2}-\mu_{2}\right)$. Also in this case, a minimum is found at $\theta_{1^{\prime}}=0$.

3. $f_{m t v M}\left(\theta_{1^{\prime}}\right)$ presents two differentiated maxima if and only if one of the two following cases applies: 
(a) $\cos \left(b_{2}-\mu_{2}\right)<\cos \left(a_{2}-\mu_{2}\right)$ and $f_{u m t v M}^{\prime}\left(\theta_{1^{\prime}} ; \lambda, \mu_{1}, \mu_{2}, \kappa_{1}, \kappa_{2}, \mu_{2}, a_{2}, b_{2}\right)$ has exactly two zero points in $\theta_{1^{\prime}} \in\left[-\frac{\pi}{2}, 0\right]$

(b) $\cos \left(b_{2}-\mu_{2}\right)>\cos \left(a_{2}-\mu_{2}\right)$ and $f_{u m t v M}^{\prime}\left(\theta_{1^{\prime}} ; \lambda, \mu_{1}, \mu_{2}, \kappa_{1}, \kappa_{2}, \mu_{2}, a_{2}, b_{2}\right)$ has exactly two zero points in $\theta_{1^{\prime}} \in\left[0, \frac{\pi}{2}\right]$

4. $f_{m t v M}\left(\theta_{1^{\prime}}\right)$ is unimodal with mode not at $\mu_{1}$ if the parameters do not match any of the above cases,

where $T\left(\lambda, \mu_{2}, \kappa_{1}, \kappa_{2}, a_{2}, b_{2}\right)$ is the test function and is defined as

$$
T\left(\lambda, \mu_{2}, \kappa_{1}, \kappa_{2}, a_{2}, b_{2}\right)=-\frac{\kappa_{1}}{\lambda^{2}}+\frac{\int_{a_{2}}^{b_{2}} \sin ^{2}\left(\theta_{2}-\mu_{2}\right) e^{\kappa_{2} \cos \left(\theta_{2}-\mu_{2}\right)} d \theta_{2}}{\int_{a_{2}}^{b_{2}} e^{\kappa_{2} \cos \left(\theta_{2}-\mu_{2}\right)} d \theta_{2}},
$$

and $f_{u m t v M}^{\prime}\left(\theta_{1^{\prime}} ; \lambda, \mu_{1}, \mu_{2}, \kappa_{1}, \kappa_{2}, \mu_{2}, a_{2}, b_{2}\right)$ is the unnormalized truncated marginal von Mises derivative function.

\section{Simulation}

In this section we experimentally test the behavior and properties of the univariate and bivariate truncated distributions. We have implemented an acceptancerejection algorithm for simulation and maximum likelihood estimation by optimization over the likelihood function for both cases. We will confirm the expected effect of manipulating the parameters of the distribution by conducting different samplings and estimation operations. In the bivariate case, our studies are focus on the effect of the $\lambda$ and truncation parameters.

\subsection{Univariate case simulation}

For the univariate case:

1. In our first simulation, we sampled 20000 points from the univariate distribution $f_{t v M}(\theta ; \pi, 2,1,5)$ (Figure $\left.4 \mathrm{~A}\right)$. This resulted in a nearly symmetrical distribution, where the sample mean $\hat{\theta}=3.1299 \approx \pi$. In this case, $\mathbb{O}_{a, b}$ selects most of the area of the original von Mises distribution. Maximizing the log-likelihood function (3) yielded values $\hat{\mu}=3.1412, \hat{\kappa}=1.9932, \hat{a}=1.0014$

and $\hat{b}=4.9940$. These values are indicative of a successful estimation of the parameters with errors roughly around $10^{-3}$. 

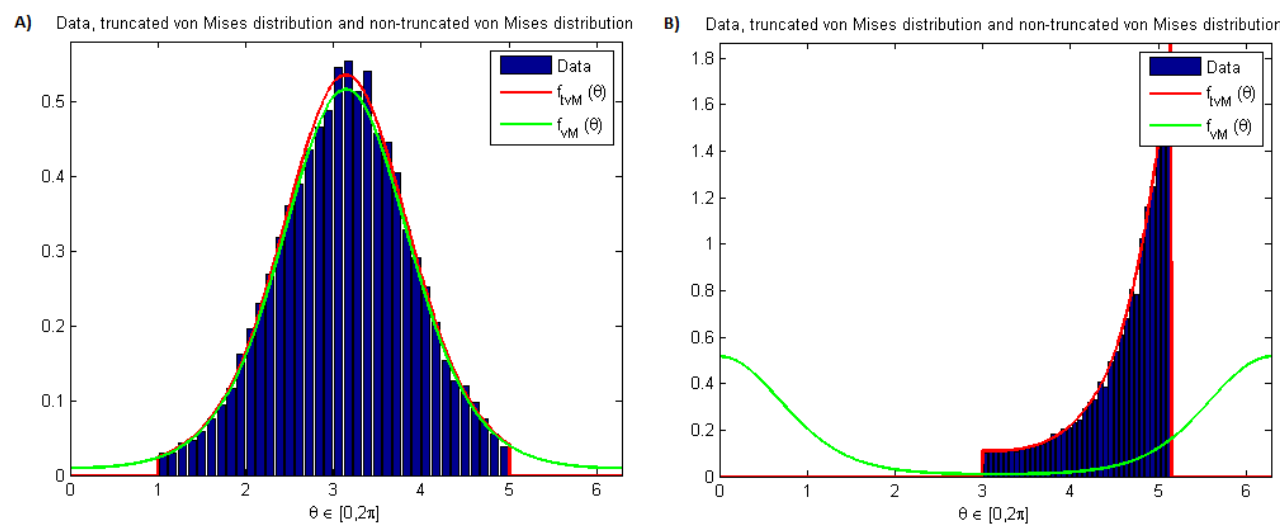

Figure 4: Simulation of two truncated von Mises distributions. The data were grouped in same-length value intervals in order to ilustrate its relative frequency.

2. We sampled 20000 points from the univariate distribution $f_{t v M}(\theta ; 0,2,3, \pi+$ 2) (Figure 4B). Its shape is highly asymmetrical with truncation parameters that satisfy $\mu+\pi \in \mathbb{O}_{a, b}$ and $\mu \notin \mathbb{O}_{a, b}$. We chose this case because it was apparently troublesome to estimate and identify as a truncated von Mises distribution, i.e., the differences w.r.t. the original von Mises distribution are very noticeable. We find that the sample mean $\hat{\theta}=4.6335$ clearly differs from the location parameter. Maximizing the likelihood function yielded parameter values $\hat{\mu}=6.3044$ (which, given the periodicity of the function, could be also considered $\mu=6.3044-2 \pi \approx 0$ ), $\hat{\kappa}=2.0446, \hat{a}=3.0004$, $\hat{b}=5.1415$.

3. We tested the distribution for relatively high concentrations by sampling 20000 points from the univariate distribution $f_{t v M}(\theta ; \pi, 15,0.5, \pi)$ (Figure $5)$. We can see how the truncation parameters retain slightly less than half of the area of the non-truncated distribution, this in turn causes the density to be slightly greater than twice the non-truncated density in its possitive support. Maximizing the log-likelihood function yielded parameter values $\hat{\mu}=3.1386, \hat{\kappa}=15.2597, \hat{a}=2.0877, \hat{b}=3.1416$. 


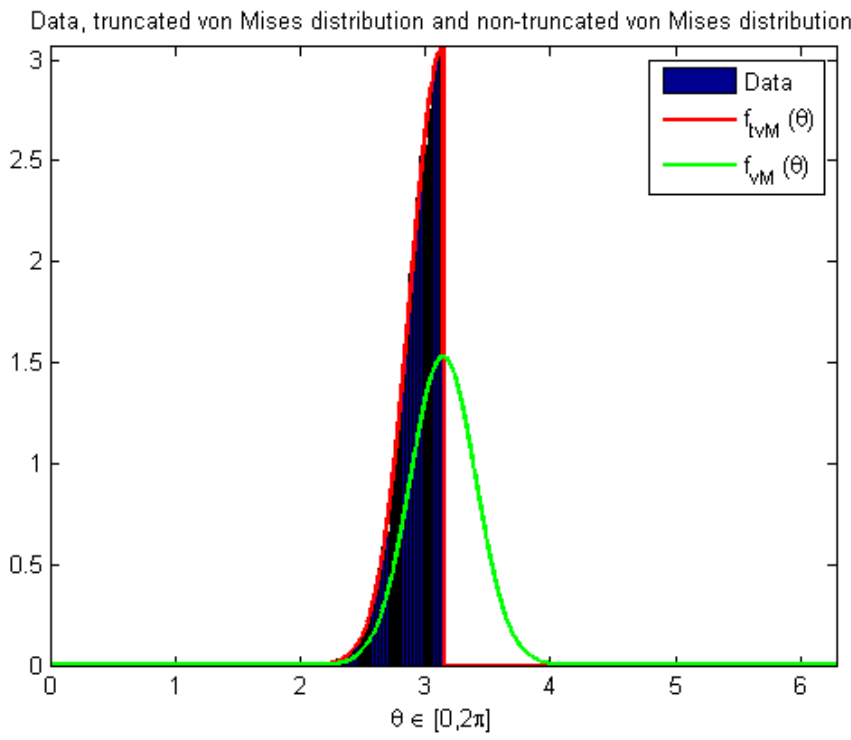

Figure 5: Simulation of the truncated von Mises distribution of the third study.

\subsection{Bivariate case simulation}

For the bivariate case:

1. We sampled 20000 points from the bivariate distribution $f_{b t v M}\left(\theta_{1}, \theta_{2} ; 1,2, \pi, 3,1,0,4,3,6\right)$ (Figure 6 ). From a visual inspection, this has a low lambda parameter and shows unimodality. The truncation parameters for this case more than halve the volume by selecting the positive support in the $\mathbb{O}_{a_{1}, b_{1}} \times \mathbb{O}_{a_{2}, b_{2}}$ region. Thus we find, for example, that it is possible to build a truncated bivariate von Mises distribution that shows only one maximum whereas its associated non-truncated distribution produces a bi-maximal distribution. The maximization of the log-likelihood function yielded parameter values $\hat{\lambda}=0.7579, \hat{\mu_{1}}=2.0540, \hat{\mu_{2}}=3.2657, \hat{\kappa}_{1}=$ $2.9753, \hat{\kappa}_{2}=1.0516, \hat{a}_{1}=0.0353, \hat{b}_{1}=3.9846, \hat{a}_{2}=3.0006, \hat{b}_{2}=5.9910$. This is a lowery quality approximation with an error of around $10^{-1}$. 

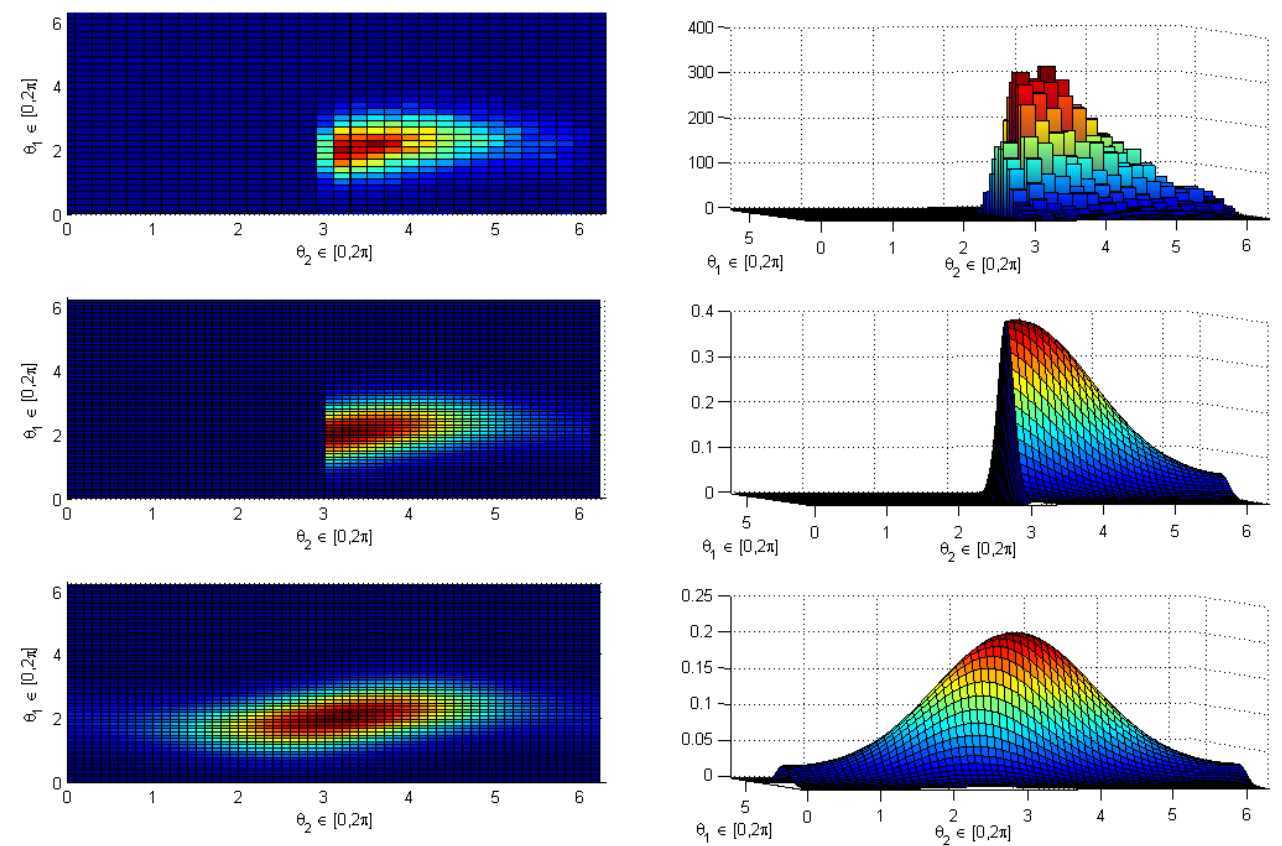

Figure 6: Simulation of a bivariate truncated von Mises distribution with parameters $\lambda=1, \mu_{1}=2, \mu_{2}=\pi, \kappa_{1}=3, \kappa_{2}=1, a_{1}=0, b_{1}=4, a_{2}=3, b_{2}=6$ from two different perspectives. The top plot corresponds to the data grouped by equal-length $\times$ equallength value square areas in order to clearly ilustrate its relative frequency, the middle plot corresponds to the truncated bivariate von Mises distribution and the bottom plot corresponds to the associated bivariate distribution.

2. The next study was similar but reformulated the parameters to accont for the case when truncation omits one of the maxima of an otherwise bimodal distribution. To be precise, we studied 20000 points sampled from $f_{b t v M}\left(\theta_{1}, \theta_{2} ; 5,2, \pi, 4,2,2.5,4,3,6\right)$ (Figure 7$)$, where the truncation parameters $a_{1}$ and $a_{2}$ were altered in order to select only part of one of the maxima. Notice also that in this case a significantly higher $\lambda$ parameter was used in order to achieve bimodality. Maximizing the log-likelihood function for this case yielded parameter values $\hat{\lambda}=4.9199, \hat{\mu}_{1}=2.0093, \hat{\mu}_{2}=3.0476, \hat{\kappa}_{1}=$ $4.0643, \hat{\kappa}_{2}=1.48, \hat{a}_{1}=2.5008, \hat{b}_{1}=3.9788, \hat{a}_{2}=3.0014, \hat{b}_{2}=5.9947$ with a similar error of around $10^{-1}$. 

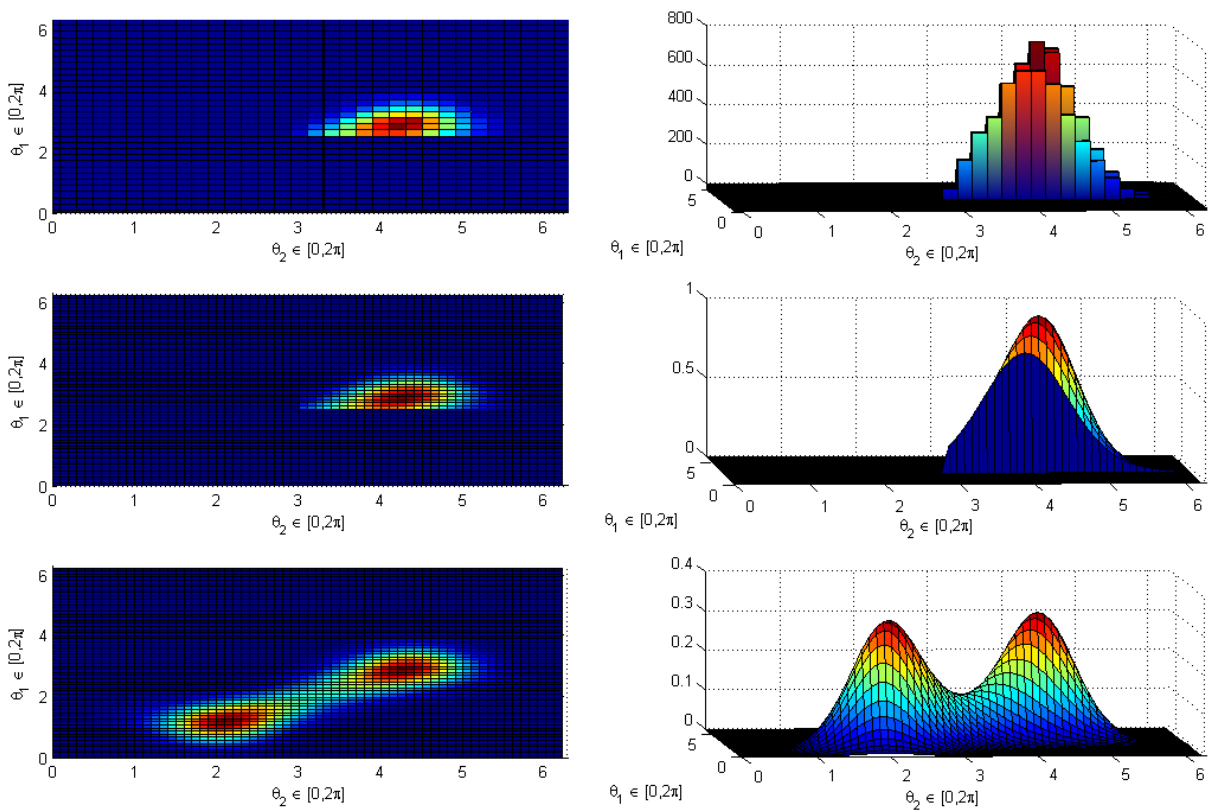

Figure 7: Simulation of a bivariate truncated von Mises distribution with parameters $\lambda=5, \mu_{1}=2, \mu_{2}=\pi, \kappa_{1}=4, \kappa_{2}=2, a_{1}=2.5, b_{1}=4, a_{2}=3, b_{2}=6$ from two different perspectives.

\section{$5 \quad$ Real data applications}

We further illustrate the truncated case by analyzing the data obtained by Bowyer and Danson. (2005) for the univariate case and Murzin AG (1995) for the bivariate case.

\subsection{Leaf angle inclination}

The data in Bowyer and Danson. (2005) was collected during a safari along the Kalahari Transect, southwest Botswana in 2001. It contains measurements of leaf inclination angles of four different woody plant species (Acacia erioloba, Grewia flava, Acacia leuderitzii and Acacia mellifera) across three different regions (Mabuasehube, Tsabong and Tshane). The measurements were taken using a clinometer.

In order to formally test the goodness-of-fit of the estimated distributions, 
we transform the data by means of the random variable $U=2 \pi \frac{[I(\theta, \mu, \kappa)-I(a, \mu, \kappa)]}{\int_{a}^{b} e^{\kappa \cos (\theta-\mu)} d \theta}$ $\bmod 2 \pi$ that is applied over the sorted sample $\theta_{1}, \ldots, \theta_{n}$. If the data distribute according to the truncated von Mises distribution, then the above random variable has a uniform distribution. As shown in Mardia and Jupp (2000), the modified Rayleigh statistic $S^{*}=\left(1-\frac{1}{2 n}\right) 2 n R^{2}+\frac{n R^{4}}{2}$, where $n$ is the sample size and $R$ the mean resultant length, distributes as a $\chi_{2}^{2}$ distribution.

1. For the first study, the whole dataset containing a total of 741 samples was observed without further regard for region or type of plant (Table 1, Figure 8). A visual inspection of the plot clearly shows that the truncated von Mises distribution performs better. Formally, for the truncated case, $S^{*}=2.8887$, which corresponds to a significance level of between 0.2 and 0.3 and acceptance of this distribution hypothesis. For the non-truncated case, $S^{*}=25.5028$, with is a clear rejection with a significance level of less than 0.001. From these results we conclude that the truncated distribution is significantly better for these data. Truncation parameters conform the circular interval $\mathbb{O}_{0, \frac{\pi}{2}}$, which indicates no angle greater than $90^{\circ}$ was measured in this study.

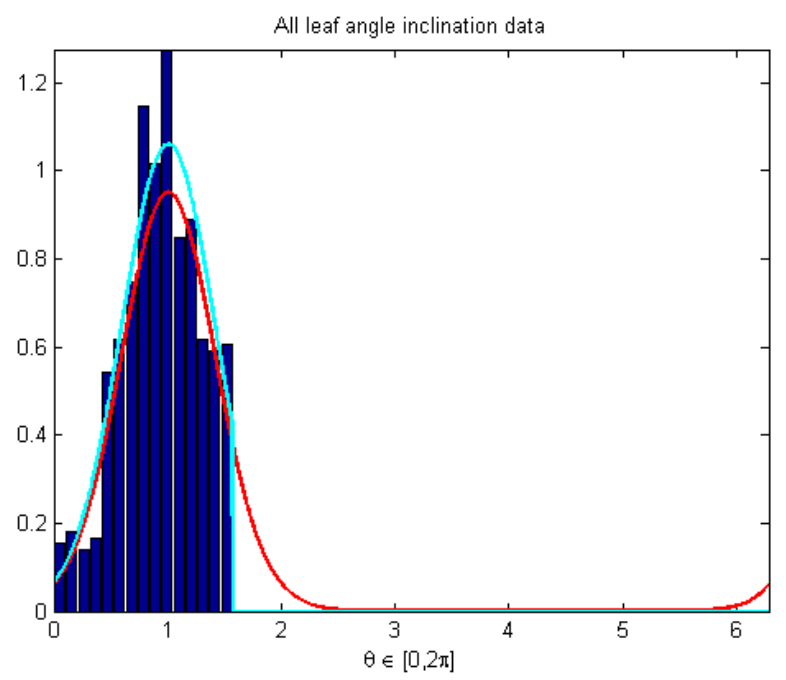

Figure 8: The study distribution and data representation of the entire dataset. The estimated truncated von Mises distribution (lighter line) clearly has higher density values than its associated von Mises distribution (darker line). The data are grouped by value intervals in order to observe its relative frequency (bars). 
Table 1: Parameter values obtained after conducting the first study

\begin{tabular}{cccccc} 
& $\mu$ & $\kappa$ & $a$ & $b$ & No.Samples \\
\hline All data & 1.0063 & 5.9602 & 0 & 1.5708 & 741
\end{tabular}

2. For the second study, we grouped the data by plant types without regards for region. This yielded four different distributions. A visual inspection shows that the univariate distributions are clearly better than the nontruncated von Mises distribution at describing the resulting data (Table 3, Figure 9), except for the case of $A$. erioloba. The goodness-of-fit tests (Table 2) revealed that the non-truncated distribution is rejected in all cases but in A.erioloba, whereas the truncated distribution hypothesis was more strongly accepted than that of the non-truncated distribution in all cases. Thus we can conclude that, for this study, the truncated distribution models the data better.

Table 2: Modified Rayleigh statistic values for the second study

\begin{tabular}{ccc} 
& Truncated von Mises $S^{*}$ & Non-truncated von Mises $S^{*}$ \\
\hline A. erioloba & 3.014 & 3.5534 \\
Grewia flava & 0.0038 & 20.6273 \\
A. leuderitzii & 2.6073 & 10.1990 \\
A. mellifera & 1.3157 & 7.3046
\end{tabular}

Truncation parameters were consistently found to be in $\mathbb{O}_{0, \frac{\pi}{2}}$ except for A.erioloba, which also presented a significantly higher concentration parameter than in any of the other estimations. The irregularities in A.erioloba could partially be explained by the small sample size, which causes the estimations to be less reliable. More data would be needed to clarify the current results. On the whole, the remaining studies show few variations in the location-concentration parameters, which closely resemble the ones obtained in the first study. 

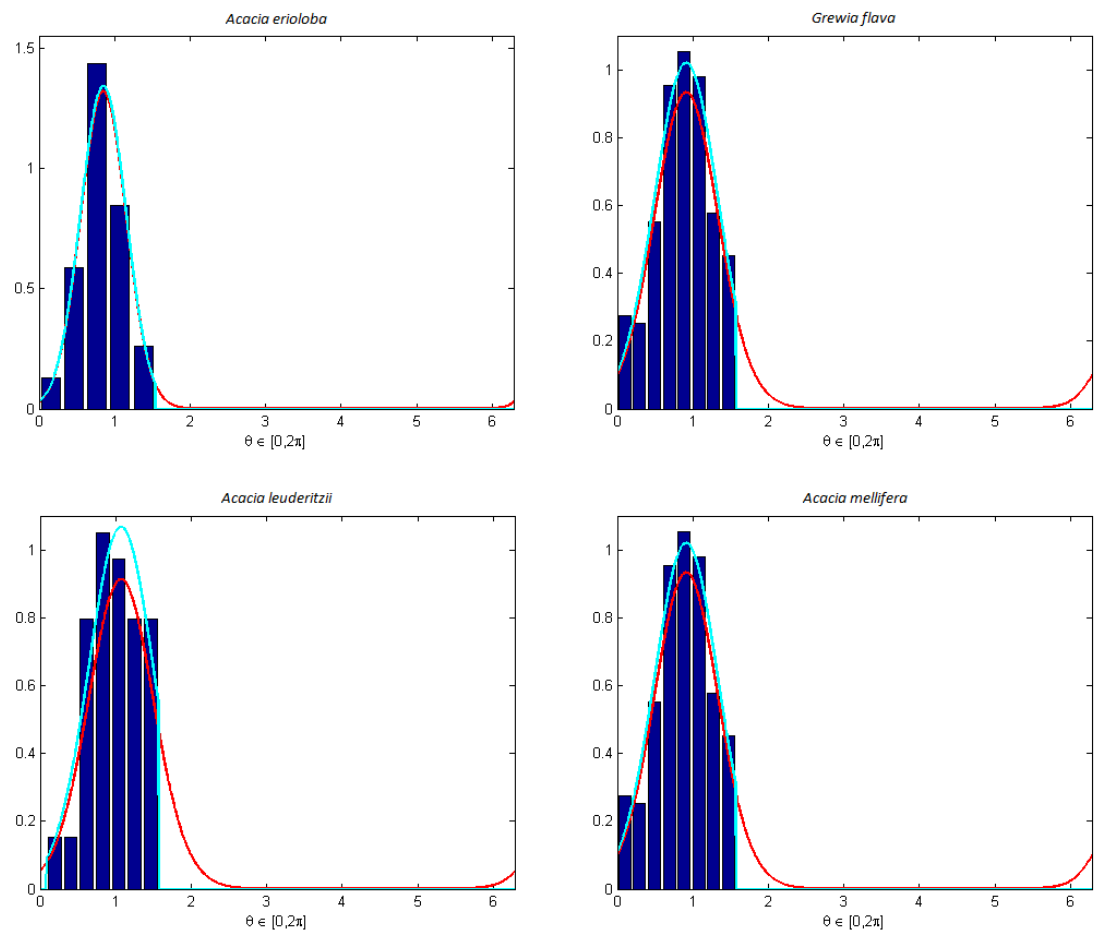

Figure 9: Studies of each type of plant.

Table 3: Parameter values yielded after conducting the second study

\begin{tabular}{cccccc} 
& $\mu$ & $\kappa$ & $a$ & $b$ & No.Samples \\
\hline A. Erioloba & 0.8516 & 11.1894 & 0 & 1.5359 & 100 \\
Grewia flava & 1.1261 & 5.2668 & 0 & 1.5708 & 254 \\
A. Leuderitzii & 1.0706 & 5.5138 & 0 & 1.5708 & 184 \\
A. Mellifera & 0.9125 & 5.7396 & 0 & 1.5708 & 203
\end{tabular}

3. For the third study, we separately fitted univariate truncated distributions to the data for each plant in each region. Since not all plants were measured in all regions, this procedure produced eight different univariate truncated von Mises estimations. The distributions are generally observed to clearly differ from their associated non-truncated von Mises distribution, except in the first of the eight plots (Table 5, Figure 10). The goodness-of-fit tests (Table 4) are also consistent with previous studies. All truncated von Mises hypotheses were accepted, while around half of the non-truncated 
distributions were rejected. Also, acceptance is stronger for the truncated case in all cases where both tested distributions were accepted. Thus, there is a strong suggestion that the truncated von Mises distribution properly models the underlying behavior that yielded the data.

Table 4: Parameter values yielded after conducting the third study

\begin{tabular}{ccc} 
& Truncated von Mises $S^{*}$ & Non-truncated von Mises $S^{*}$ \\
\hline A. erioloba, Mabuasehube & 3.014 & 3.5534 \\
Grewia flava, Mabuasehube & 1.1543 & 8.9599 \\
A. leuderitzii, Tsabong & 2.0981 & 7.3115 \\
Grewia flava, Tsabong & 0.2050 & 3.8702 \\
A. mellifera, Tsabong & 0.1199 & 4.2131 \\
Grewia flava(2), Tsabong & 0.1165 & 9.7290 \\
A. leuderitzii, Tshane & 0.7002 & 2.8717 \\
A. mellifera, Tshane & 1.0525 & 10.2656
\end{tabular}

For this study, each distribution was estimated from a relatively small sample size ranging from 50 to 104 samples, which may have caused estimations to be less precise than desired. The concentration parameter shows the highest variability across the different cases (from 4.4078 to 11.1894 across the whole study or even from 4.8340 to 7.4245 in the case of A. leuderitzii). With more data it might be possible to distinguish if the variations in the concentration parameter are clearly influenced by the region of the plant species or the small sample size. Regarding the location parameter, there are few variations in the parameter value on the whole, A. mellifera being the species that experienced the highest variations w.r.t. one of the measurements in the first study. Truncation parameters remained consistently within the $\mathbb{O}_{0, \frac{\pi}{2}}$ interval. 

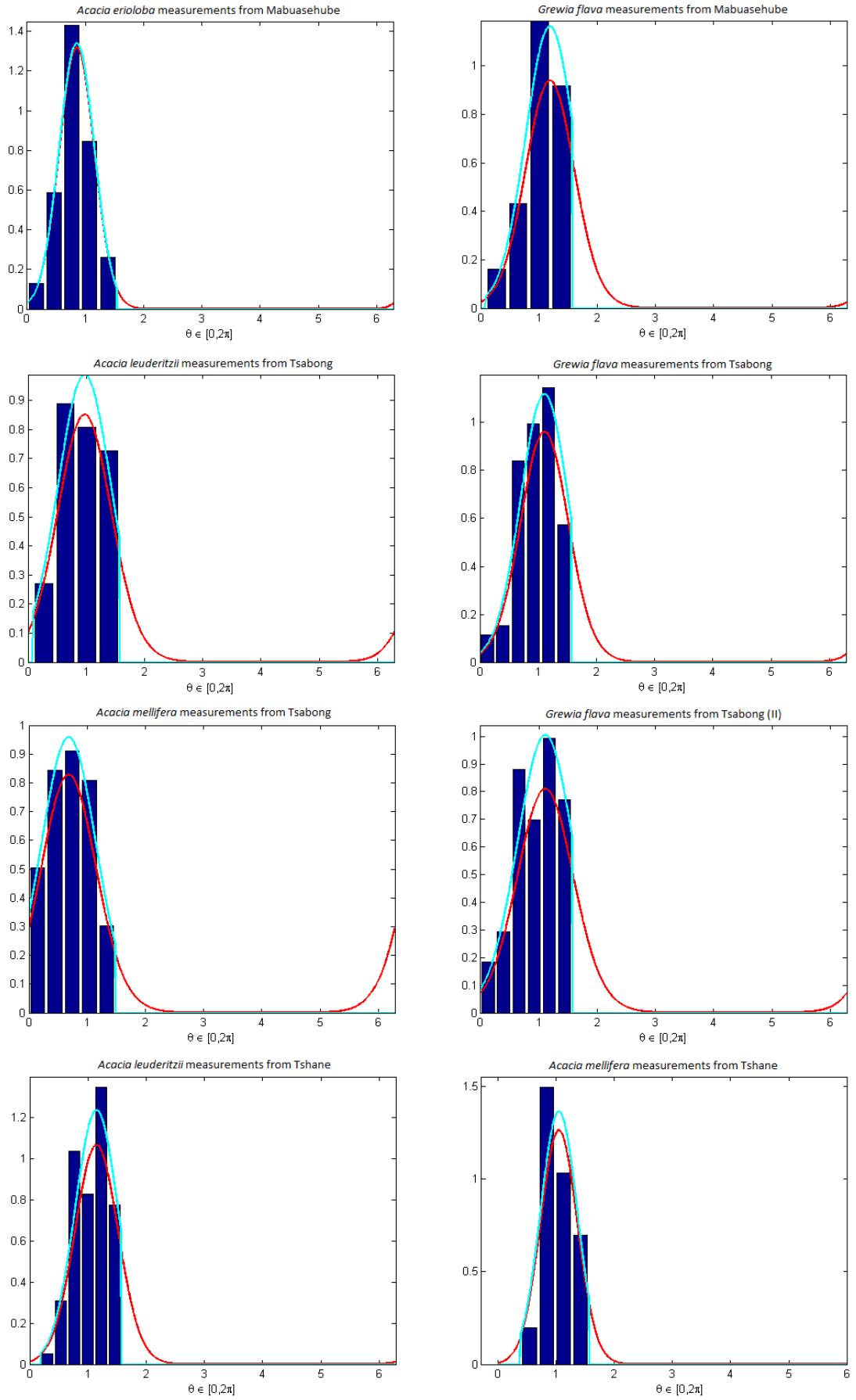

Figure 10: Studies of each type of plant in each region. 
Table 5: Parameter values yielded after conducting the third study

\begin{tabular}{cccccc} 
& $\mu$ & $\kappa$ & $a$ & $b$ & No.Samples \\
\hline A. erioloba, Mabuasehube & 0.8516 & 11.1894 & 0 & 1.5359 & 100 \\
Grewia flava, Mabuasehube & 1.1882 & 5.8142 & 0.0873 & 1.5708 & 50 \\
A. leuderitzii, Tsabong & 0.9712 & 4.8340 & 0.0873 & 1.5708 & 100 \\
Grewia flava, Tsabong & 1.1082 & 6.0832 & 0 & 1.5708 & 100 \\
A. mellifera, Tsabong & 0.6844 & 4.5884 & 0 & 1.4835 & 100 \\
Grewia flava (2), Tsabong & 1.1091 & 4.4078 & 0 & 1.5708 & 104 \\
A. leuderitzii, Tshane & 1.1474 & 7.4245 & 0.1920 & 1.5708 & 84 \\
A. mellifera, Tshane & 1.0525 & 10.2656 & 0.4014 & 1.5708 & 103
\end{tabular}

\subsection{Pairs of dihedral angles in protein chains}

This study consists of a single estimation for the bivariate case. The dataset corresponds to the spatial coordinates of the $\beta$-class atom in the SCOP-ASTRAL database (Murzin AG (1995)). Specifically, we extract the data on the position of the alpha-carbon atoms within a protein chain. The data are properly transformed from a set of coordinates to a set of pairs of angles $(\theta, \phi)$ that account for the relative angular deviation of the next node in the protein chain has the previous part of the chain. After applying this procedure, the resulting sample contained 288610 observations.

The data have two clearly distinguishable modes that are separated by an area of low or zero density. This area is known to be empty in protein chains, which our model will assume by fixing the truncation parameters at $\mathbb{O}_{4.8,2.2}$. For this dataset, the bivariate truncated distribution is capable of capturing bimodality and defining an area of empty density as desired, and the parameter estimation by the maximum likelihood method provided a model that clearly exhibited theseh features (Figure 11). 

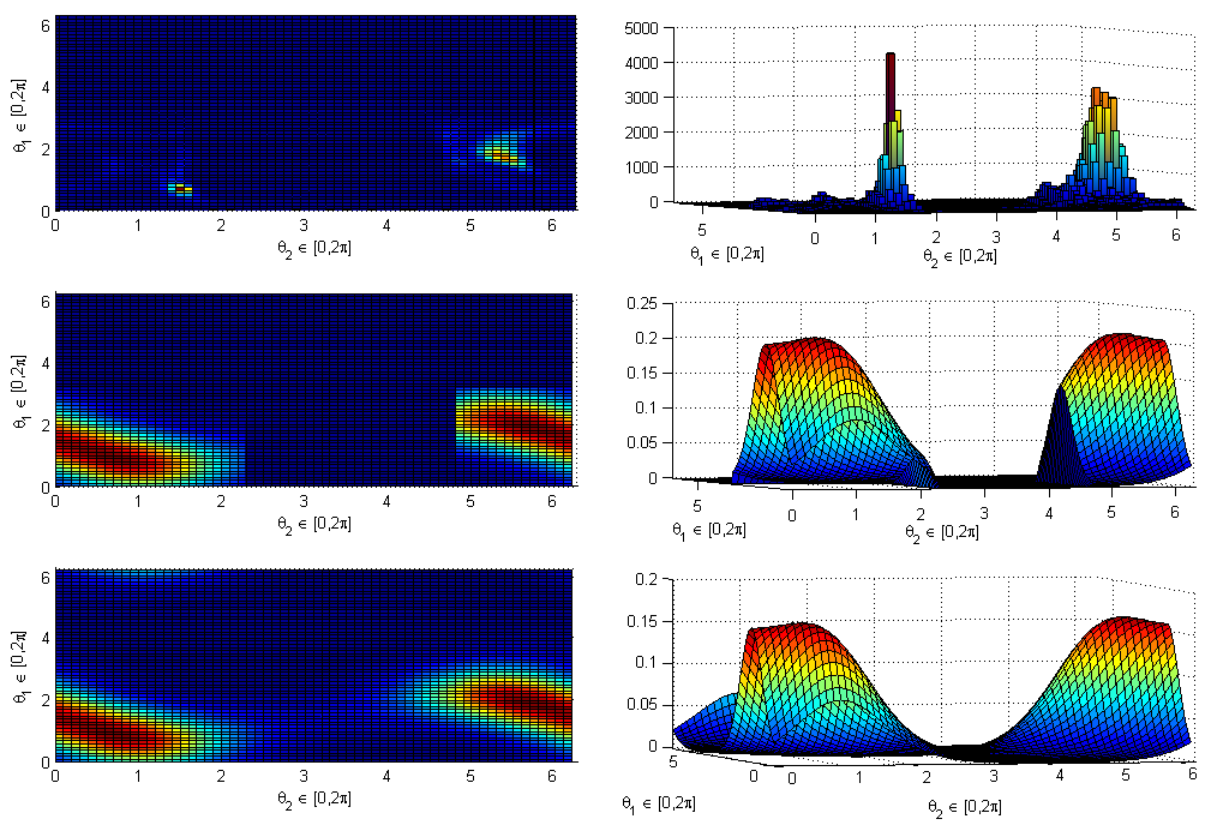

Figure 11: Estimated truncated von Mises distribution for the entire dataset. This distribution corresponds to the parameter values in Table 4. The top plot corresponds to a data frequency plot, the middle plot corresponds to the estimated bivariate truncated von Mises distribution and the bottom plot corresponds to its associated non-truncated distribution.

Table 6: Parameter values yielded after conducting the study on the protein chain dataset

\begin{tabular}{cccccccc}
$\lambda$ & $\mu_{1}$ & $\mu_{2}$ & $\kappa_{1}$ & $\kappa_{2}$ & $a_{1}$ & $b_{1}$ & No.Samples \\
\hline-1.93333 & 1.3859 & 0 & 2.1477 & 1.1213 & 0.0023 & 3.1363 & 288,610
\end{tabular}

In this case (Table 6 ), there seems to be a consistent inverse correlation between the pairs of angles as shown by the value of the lambda parameter. This plays a role in creating the observed bi-modality.

We conclude that the truncated von Mises distribution can be used to effectively model, simulate and summarize data about real-world phenomena and may be applied in any experiment whose outcome can be expressed in angular values. 


\section{Summary and conclusions}

In this article we developed the theoretical framework of the univariate and the bivariate truncated von Mises distribution. To do this, we gave

1. The definition of a truncated von Mises distribution in the circle $\mathbb{O}$. The circular distribution is defined by means of the $\mathbb{O}$ subset, as the periodicity and properties of the circlehave to be naturally acknowledged for. If the $a, b$ parameters were to not include $0^{\circ}$ (our reference point) or $f_{t v M}(0 ; \mu, \kappa, a, b)=$ 0 , then linear definitions for the truncation parameters could be used. Therefore, $\mathbb{O}_{a, b}$ becomes $[a, b]$ with the restriction of $a<b$ and maximum likelihood estimators $\hat{a}, \hat{b}$ become $\hat{a}=\min \left\{\theta_{1}, \ldots, \theta_{n}\right\}, \hat{b}=\max \left\{\theta_{1}, \ldots, \theta_{n}\right\}$ respectively. The extension for the bivariate case is trivial.

2. The successfully determined expressions of the maximum likelihood estimators. For both univariate and bivariate cases, solely sample-dependent maximum likelihood estimators of the truncation parameters were found, while the other parameters showed interdependency.

3. The resulting moments of the univariate case and existing interrelationships.

4. The properties of both bivariate and univariate cases, especially the results concerning the additional manipulability and possible shapes of the distribution when modifying the truncation parameters, that is, a distribution can be made to be strictly increasing or strictly decreasing symmetrical or non-symmetrical function, or to concentrate its positive support in an arbitrarily short sub-interval.

5. The bivariate case and studies of the shape and behavior of marginal and conditional distributions. We determined that every conditional truncated von Mises distribution is a univariate truncated von Mises distribution. For the case of the marginal distribution, we concluded that only for parameter $\lambda=0$ does the distribution behave like a truncated univariate von Mises distribution. When $\lambda \neq 0$, the resultant marginal distribution is a potentially bi-maximal not a von Mises distribution. We obtained four different cases in order to characterize all the different truncated marginal von Mises 
behaviors. To be precise, when the marginal distribution is bi-maximal, it exhibits either one of two or two global maxima and the minimum value is not at $\mu_{1}$ if truncation parameters $a_{2}$ and $b_{2}$ are not symmetrical w.r.t. $\mu_{2}$, and it is if they are. When the marginal distribution is unimodal, the maximum value is not at $\mu_{1}$ if truncation parameters $a_{2}$ and $b_{2}$ are not symmetrical w.r.t. $\mu_{2}$, and it is if they are. This covers all possible shapes or behaviors.

\section{SUPPLEMENTARY MATERIAL}

Appendix All proofs of introduced lemmas and theorems (.pdf file). 


\section{References}

Abramowitz, M., and Stegun, I. (1964), Handbook of Mathematical Functions: With Formulas, Graphs, and Mathematical Tables, Applied Mathematics Series Dover Publications.

Bistrian, D. A., and Iakob, M. (2008), "One-dimensional truncated von Mises distribution in data modeling," Annals of Faculty of Engineering Hunedoara, tome VI (year 2008), fascicule 3, .

Bowyer, P., N. M. T., and Danson., F. M. (2005), "SAFARI 2000 Canopy Structural Measurements, Kalahari Transect, Wet Season 2001. Data set.,".

Jupp, P. E., and Mardia, K. V. (1989), "A unified view of the theory of directional statistics, 1975-1988," International Statistical Review, 57(3), 261-294.

Lopez-Cruz, P., Bielza, C., and Larrañaga, P. (2013), "Directional naive Bayes classifiers," Pattern Analysis and Applications, pp. 1-22.

Mardia, K., and Jupp, P. (2000), Directional Statistics, Wiley Series in Probability and Statistics.

Mardia, K. V., Hughes, G., Taylor, C. C., and Singh, H. (2008), "A multivariate von Mises distribution with applications to bioinformatics," Canadian Journal of Statistics, 36, 99-109.

Mardia, K. V., and Voss, J. (2011), "Some fundamental properties of a multivariate von Mises distribution," ArXiv e-prints, .

Murzin AG, Brenner SE, H. T. C. C. (1995), "SCOP: a structural classification of proteins database for the investigation of sequences and structures,". 
Singh, H. (2002), "Probabilistic model for two dependent circular variables," Biometrika, 89(3), 719-723.

Computational Intelligence Group, Department of Artificial Intelligence, Universidad Politécnica de Madrid

E-mail: pablo.fernandezgonz@fi.upm.es

Department of Artificial Intelligence, Universidad Politécnica de Madrid

E-mail: mcbielza@fi.upm.es

Department of Artificial Intelligence, Universidad Politécnica de Madrid

E-mail: plarranaga@fi.upm.es 